\title{
Acoustic leakage in electromagnetic waveguides made from piezoelectric materials
}

\author{
Jiashi Yang \\ Key Laboratory of Low Dimensional Materials and Application Technology, \\ (Xiangtan University), Ministry of Education, Xiangtan, Hunan 411105, China \\ A.-K. Soh ${ }^{\mathrm{a})}$ and Xiaohong Chen \\ Department of Mechanical Engineering, The University of Hong Kong, Pokfulam Road, \\ Hong Kong, China
}

(Received 28 November 2006; accepted 19 January 2007; published online 29 March 2007)

\begin{abstract}
We study the propagation of coupled acoustic and electromagnetic waves in a piezoelectric plate waveguide embedded in two half-spaces of another dielectric material. It is shown that certain waves are guided electromagnetically but not so acoustically and that these waves are effectively damped waves with acoustic leakage of energy. An estimate of the acoustic radiation damping is given. (C) 2007 American Institute of Physics. [DOI: 10.1063/1.2712157]
\end{abstract}

Design of electromagnetic (EM) waveguides and resonators has been routinely performed using Maxwell equations. ${ }^{1}$ In fact, some of the materials used for dielectric waveguides and resonators have piezoelectric coupling. These devices are often mounted on substrates of other materials with acoustic interaction. If the device material has piezoelectric coupling, the EM waves in the device are accompanied by acoustic waves interacting with the substrate. There is a possibility of acoustic leakage or radiation of energy in an EM wave device and little is known about the effect. In this short paper we examine this possible phenomenon. To describe coupled acoustic and EM wave phenomena in a piezoelectric material, a theory called piezoelectromagnetism ${ }^{2-4}$ is needed. Recently, a piezoelectromagnetic solution has been obtained for coupled elastic and EM waves propagating in a piezoelectric plate waveguide embedded in two half-spaces of another material. ${ }^{5}$ The solution was used to calculate waves that are guided both acoustically and electromagnetically, but the possibility of acoustic leakage of guided EM waves was not explored. We examined this possibility below based on the results of Ref. 5. Consider a plate of polarized ceramics between two half-spaces (see Fig. 1) of another polarized ceramics. For ceramics poled in the $x_{3}$ direction, there exist anti-plane or transverse magnetic modes ${ }^{5}$ propagating in the $x_{1}$ direction with a factor of $\cos \left(\xi x_{1}-\omega t\right)$ and only one displacement component $u_{3}$ as well as one magnetic field component $H_{3}$. The modes can be separated into symmetric and anti-symmetric according to whether $u_{3}$ is an even or odd function of $x_{2}$. The corresponding dispersion relations are

$$
\begin{gathered}
\left(\frac{\beta_{A}}{\varepsilon_{A}} \tanh \beta_{B} h+\frac{\beta_{B}}{\varepsilon_{B}}\right)\left(\bar{c}_{A} \alpha_{A}-\bar{c}_{B} \alpha_{B} \tan \alpha_{B} h\right) \\
=\left(\frac{e_{A}}{\varepsilon_{A}}-\frac{e_{B}}{\varepsilon_{B}}\right)^{2} \xi^{2} \tanh \beta_{B} h,
\end{gathered}
$$

\footnotetext{
${ }^{a)}$ Author to whom correspondence should be addressed; electronic mail: aksoh@hkucc.hku.hk
}

$$
\begin{aligned}
& \left(\frac{\beta_{A}}{\varepsilon_{A}} \operatorname{coth} \beta_{B} h+\frac{\beta_{B}}{\varepsilon_{B}}\right)\left(\bar{c}_{A} \alpha_{A}+\bar{c}_{B} \alpha_{B} \cot \alpha_{B} h\right) \\
& \quad=\left(\frac{e_{A}}{\varepsilon_{A}}-\frac{e_{B}}{\varepsilon_{B}}\right)^{2} \xi^{2} \operatorname{coth} \beta_{B} h .
\end{aligned}
$$

where

$$
\begin{aligned}
& \alpha_{A}^{2}=\xi^{2}-\rho_{A} \omega^{2} / \bar{c}_{A}=\xi^{2}\left(1-\frac{v^{2}}{v_{A}^{2}}\right), \\
& \beta_{A}^{2}=\xi^{2}-\varepsilon_{A} \mu_{0} \omega^{2}=\xi^{2}\left(1-\frac{v^{2}}{c_{A}^{2}}\right)>0, \\
& v^{2}=\frac{\omega^{2}}{\xi^{2}}, \quad v_{A}^{2}=\frac{\bar{c}_{A}}{\rho_{A}}, \quad c_{A}^{2}=\frac{1}{\varepsilon_{A} \mu_{0}} . \\
& \alpha_{B}^{2}=\rho_{B} \omega^{2} / \bar{c}_{B}-\xi^{2}=\xi^{2}\left(\frac{v^{2}}{v_{B}^{2}}-1\right), \\
& \beta_{B}^{2}=\xi^{2}-\varepsilon_{B} \mu_{0} \omega^{2}=\xi^{2}\left(1-\frac{v^{2}}{c_{B}^{2}}\right), \\
& v_{B}^{2}=\frac{\bar{c}_{B}}{\rho_{B}}, \quad c_{B}^{2}=\frac{1}{\varepsilon_{B} \mu_{0}} .
\end{aligned}
$$

$c=c_{44}, e=e_{15}$ and $\varepsilon=\varepsilon_{11}$ are the relevant elastic, piezoelectric and dielectric constants. $\mu_{0}$ is the magnetic permeability of free space. $\rho$ is the mass density. $\bar{c}=c+e^{2} / \varepsilon$ is a piezoelectrically stiffened elastic constant. The inequality in Eq. $(3)_{2}$ is for real value of $B_{3 \mathrm{~A}}$ (only the positive root is taken) so that the waves are electromagnetically guided. Whether the waves are accoustically guided or not depends on the sign of $\alpha_{A}^{2}$.

Since the speed of light is much higher than the acoustic wave speed, from Eq. (3) it can be seen that in the range of $v_{A}<v<c_{A}, \alpha_{A}^{2}$ becomes negative or $\alpha_{A}$ becomes purely imaginary while $\beta_{A}$ is still real and positive. In this case the EM fields of the coupled waves are still guided but the 


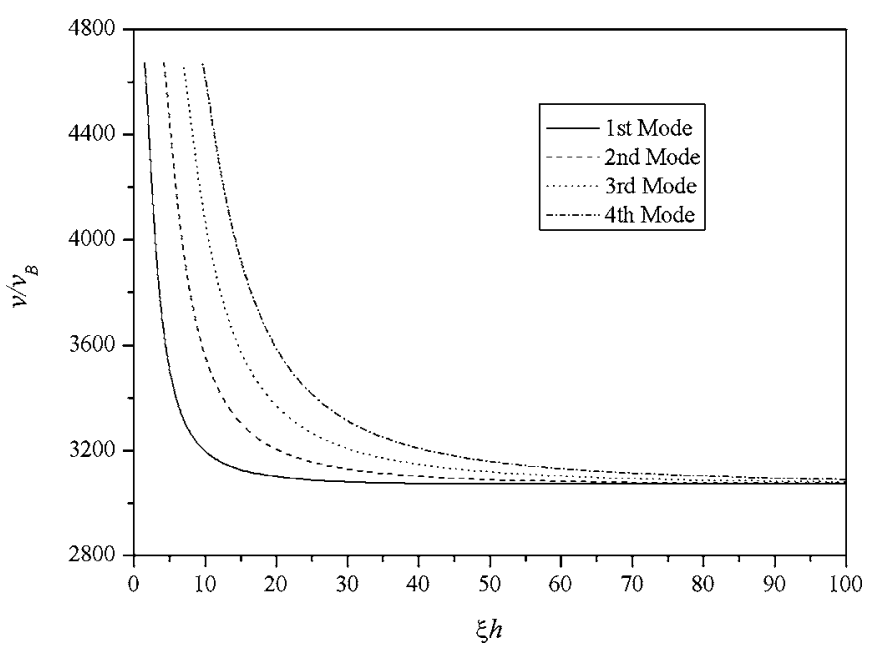

FIG. 2. Typical dispersion relations of guided em waves from Eq. (10).

acoustic fields are not. This can happen to both symmetric and antisymmetric waves. The effect of acoustic leaking is expected to be similar for symmetric and antisymmetric waves. As an example we examine the symmetric waves governed by Eq. (1) only. Consider the case in which $v_{A}$ $<v<c_{A}$. To be specific, we also limit ourselves to the case where $v>c_{B}$. Together with $v_{A}<v<c_{A}$, we consider $v$ in the range of

$$
v_{A}<c_{B}<v<c_{A} .
$$

For convenience we denote

$$
\begin{aligned}
& \alpha_{A}^{2}=\xi^{2}\left(1-\frac{v^{2}}{v_{A}^{2}}\right)=-\hat{\alpha}_{A}^{2}, \quad \alpha_{A}=i \hat{\alpha}_{A}, \\
& \beta_{B}^{2}=\xi^{2}\left(1-\frac{v^{2}}{c_{B}^{2}}\right)=-\hat{\beta}_{B}^{2}, \quad \beta_{B}=i \hat{\beta}_{B} .
\end{aligned}
$$

With Eq. (8), we can write Eq. (1) as

$$
\frac{\beta_{A}}{\varepsilon_{A}} \tan \hat{\beta}_{B} h+\frac{\hat{\beta}_{B}}{\varepsilon_{B}}=\left(\frac{e_{A}}{\varepsilon_{A}}-\frac{e_{B}}{\varepsilon_{B}}\right)^{2} \frac{\xi^{2} \tan \hat{\beta}_{B} h}{\bar{c}_{A} i \hat{\alpha}_{A}-\bar{c}_{B} \alpha_{B} \tan \alpha_{B} h},
$$

where we have also used $\tanh i Z=i \tan Z$ for a complex variable $Z$. The right-hand side of Eq. (9) is due to piezoelectric coupling which may be a small effect. We use an iteration or perturbation procedure to solve Eq. (9). As the lowest order of approximation, we neglect the right-hand side of Eq. (9) and denote the EM frequencies of the left-hand side of Eq. (9) by $\omega_{0}$,

$$
\frac{\beta_{A 0}}{\varepsilon_{A}} \tan \hat{\beta}_{B 0} h+\frac{\hat{\beta}_{B 0}}{\varepsilon_{B}} \cong 0,
$$

where

$$
\beta_{A 0}=\sqrt{\xi^{2}-\frac{\omega_{0}^{2}}{c_{A}^{2}}}, \quad \hat{\beta}_{B 0}=\sqrt{\frac{\omega_{0}^{2}}{c_{B}^{2}}-\xi^{2}} .
$$

Given a wave number $\xi$, Eq. (10) determines a series of frequencies $\omega_{0}(\xi)$ for guided EM waves in the structure. When piezoelectric coupling is considered, these EM fre-

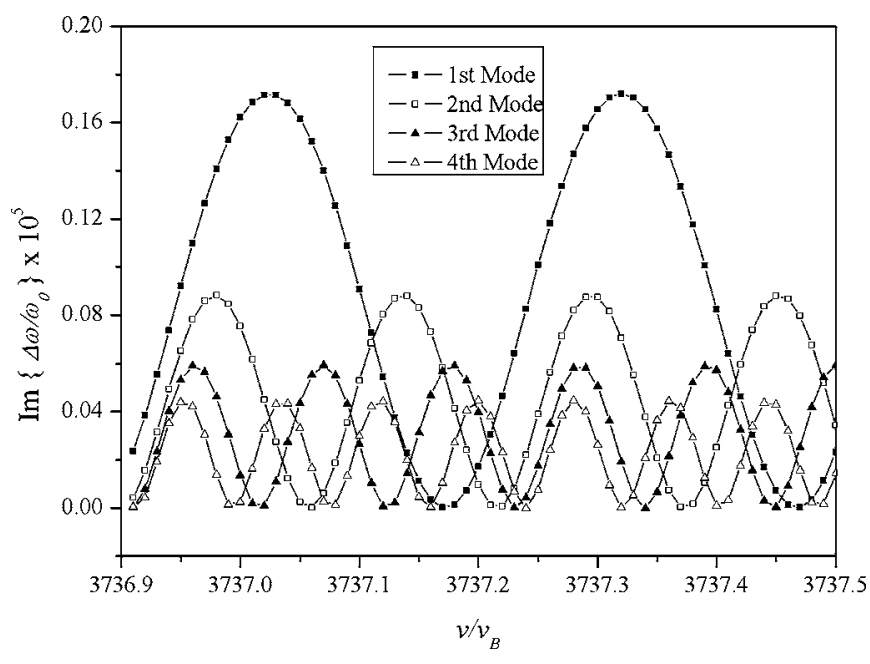

FIG. 3. Dissipation of guided em waves due to acoustic leakage.

quencies are perturbed and are determined by Eq. (9). Let the corresponding frequencies from Eq. (9) be denoted by

$$
\omega=\omega_{0}+\Delta \omega .
$$

Substituting Eq. (12) into Eq. (9), we obtain the following first-order modification of the EM frequencies due to piezoelectric coupling:

$$
\begin{aligned}
\frac{\Delta \omega}{\omega_{0}} \cong & \frac{1}{\omega_{0}^{2}}\left(\frac{e_{A}}{\varepsilon_{A}}-\frac{e_{B}}{\varepsilon_{B}}\right)^{2} \\
& \times \frac{\xi^{2} \tan \left(\hat{\beta}_{B 0} h\right)\left[-\bar{c}_{B} \alpha_{B 0} \tan \left(\alpha_{B 0} h\right)-\bar{c}_{A} i \hat{\alpha}_{A 0}\right]}{\left(\bar{c}_{A} \hat{\alpha}_{A 0}\right)^{2}+\left[\bar{c}_{B} \alpha_{B 0} \tan \left(\alpha_{B 0} h\right)\right]^{2}} \\
& \times\left(\frac{\beta_{A 0} h}{\varepsilon_{A} \hat{\beta}_{B 0} c_{B}^{2} \cos ^{2}\left(\hat{\beta}_{B 0} h\right)}-\frac{\tan \left(\hat{\beta}_{B 0} h\right)}{\varepsilon_{A} \beta_{A 0} c_{A}^{2}}\right. \\
& \left.+\frac{1}{\varepsilon_{B} \hat{\beta}_{B 0} c_{B}^{2}}\right)^{-1},
\end{aligned}
$$

where

$$
\hat{\alpha}_{A 0}=\sqrt{\frac{\omega_{0}^{2}}{v_{A}^{2}}-\xi^{2}}, \quad \alpha_{B 0}=\sqrt{\frac{\omega_{0}^{2}}{v_{B}^{2}}-\xi^{2}} .
$$

Equation (13) gives the frequency perturbation of EM waves due to piezoelectric coupling. It is a complex number. In addition to its real part representing additional dispersion, its imaginary part describes damped waves due to acoustic radiation. As a numerical example, consider PZT-4 and PZT-5H for materials $A$ and $B$ which satisfy Eq. (7). ${ }^{5}$

The first few branches of the dispersion relation for guided EM waves, i.e., solution to Eq. (10) where piezoelectric coupling is ignored, are shown in Fig. 2. These waves are clearly dispersive. For short waves with a large wave number the dispersion is small, and the wave speed of various branches seems to approach the same constant. The range of the wave speed is bounded by Eq. (7) from below and above.

When the waves represented by the dispersion relations in Fig. 2 are substituted into the right hand of Eq. (13), the imaginary part of the left-hand side of Eq. (13) which represents dissipation is shown in Fig. 3. The most basic point to 


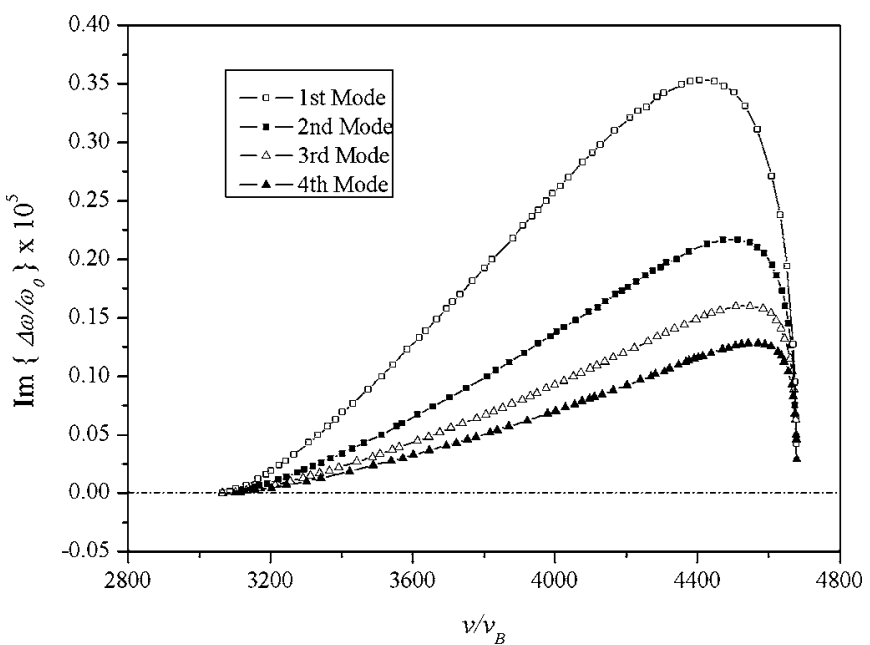

FIG. 4. Dissipation of guided em waves due to acoustic leakage (peak values).

note is that the curves in Fig. 3 are always positive indicating damped waves rather than growing waves. The decay is of the order of $10^{-5}$. The way we order the modes are such that higher-order modes have higher frequencies.

Figure 3 is for a very small range of wave speed and the curves already have quite a few oscillations. Curves corresponding to higher-order modes with higher frequencies have more oscillations. We are interested in the behavior of these curves over the entire range as bounded by (7). In Fig. 4 we only plot the peak values of the curves in Fig. 3 versus wave speed over the entire range for otherwise the figure will be too crowded. Figure 4 shows that the dissipation is sensitive to the wave speed. For the modes considered there exists a maximum dissipation at a particular wave speed. At the lower bound of the wave speed all waves approach the same speed, as shown in Fig. 2, and the corresponding dissipation also approaches a common value which is zero.

Figure 5 shows the real part of the left-hand side of Eq. (13) which represents additional dispersion induced by coupling to acoustic waves. Like in Fig. 4, only the maximum (and minimum) values of sinusoidal curves are shown in Fig. 5. The most basic qualitative difference between Figs. 4 and

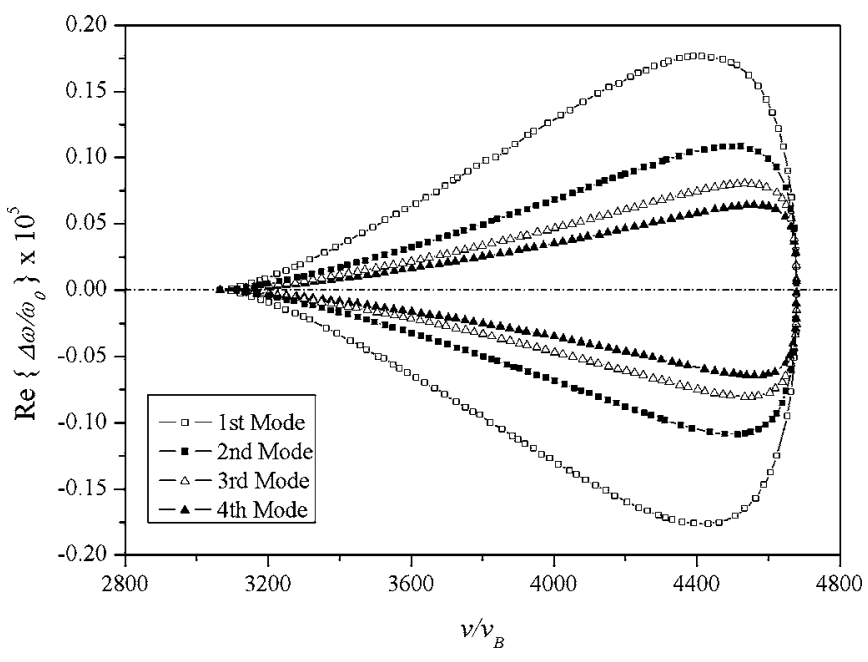

FIG. 5. Frequency shift (additional dispersion) of guided em waves due to acoustic leakage (peak values).

5 is that the maximum and minimum values of the additional dispersion in Fig. 5 are with alternating signs. The additional dispersion also has a maximum which seems to be at the same location as the maximum dissipation.

In conclusion, in piezoelectric materials, guided EM waves may be accompanied by radiating or leaking acoustic waves. This results in a radiation damping of the order of $10^{-5}$. This has implications in designing EM wave devices.

In conclusion, in piezoelectric materials, guided EM waves may be accompanied by radiating or leaking acoustic waves. This results in a radiation damping of the order of $10^{-5}$. This has implications in designing EM wave devices.

Support from the Research Grants Council of the Hong Kong Special Administrative Region, China (Project No. HKU 7195/04E) is acknowledged.

${ }^{1}$ D. Marcuse, Light Transmission Optics (Van Nostrand Reinhold, New York, 1982).

${ }^{2}$ J. J. Kyame, J. Acoust. Soc. Am. 21, 159 (1949).

${ }^{3}$ R. D. Mindlin, Int. J. Solids Struct. 9, 697 (1972).

${ }^{4}$ P. C. Y. Lee, J. Appl. Phys. 69, 7470 (1991).

${ }^{5}$ S. N. Jiang, Q. Jiang, X. F. Li, S. H. Guo, H. G. Zhou, and J. S. Yang, Int. J. Solids Struct. 43, 5799 (2006). 